\title{
Segmentation of Vessels Cluttered with Cells Using a Physics Based Model
}

\author{
Stephen J. Schmugge ${ }^{1}$, Steve Keller ${ }^{3}$, Nhat Nguyen $^{1}$, Richard Souvenir ${ }^{1}$, \\ Toan Huynh ${ }^{3}$, Mark Clemens ${ }^{2}$, and Min C. Shin ${ }^{1}$ \\ ${ }^{1}$ Department of Computer Science, University of North Carolina at Charlotte, USA \\ sjschmug@uncc.edu, nhnguye1@uncc.edu, souvenir@uncc.edu, mcshin@uncc.edu \\ ${ }^{2}$ Department of Biology, University of North Carolina at Charlotte, USA \\ mgclemen@uncc.edu \\ ${ }^{3}$ Carolinas Medical Center, Charlotte,NC, USA \\ Steven.Keller@carolinashealthcare.org, thuynh@carolinashealthcare.org
}

\begin{abstract}
Segmentation of vessels in biomedical images is important as it can provide insight into analysis of vascular morphology, topology and is required for kinetic analysis of flow velocity and vessel permeability. Intravital microscopy is a powerful tool as it enables in vivo imaging of both vasculature and circulating cells. However, the analysis of vasculature in those images is difficult due to the presence of cells and their image gradient. In this paper, we provide a novel method of segmenting vessels with a high level of cell related clutter. A set of virtual point pairs ("vessel probes") are moved reacting to forces including Vessel Vector Flow $(\mathrm{VVF})$ and Vessel Boundary Vector Flow (VBVF) forces. Incorporating the cell detection, the VVF force attracts the probes toward the vessel, while the VBVF force attracts the virtual points of the probes to localize the vessel boundary without being distracted by the image features of the cells. The vessel probes are moved according to Newtonian Physics reacting to the net of forces applied on them. We demonstrate the results on a set of five real in vivo images of liver vasculature cluttered by white blood cells. When compared against the ground truth prepared by the technician, the Root Mean Squared Error (RMSE) of segmentation with VVF and VBVF was $55 \%$ lower than the method without VVF and VBVF.
\end{abstract}

\section{Introduction}

The segmentation of vasculature in biomedical images can provide insight into analysis of vascular morphology, topology and is required for kinetic analysis of flow velocity and vessel permeability. The measurements of diameter, tortuosity, and bifurcations are often manually measured for further analysis. When the number of vessels to be analyzed is large, the automation facilitates analysis of large datasets (liver lobules can have extensive microvascular network) and decreases technician related bias.

A number of vessel segmentation algorithm has been proposed and are mostly in the domain of retina images. In this work, we developed a vessel segmentation 


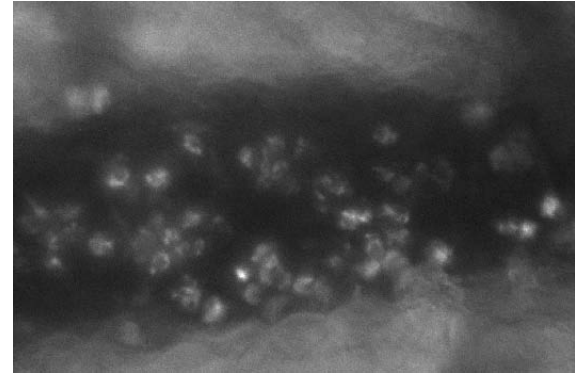

(a) intravital microscopy image

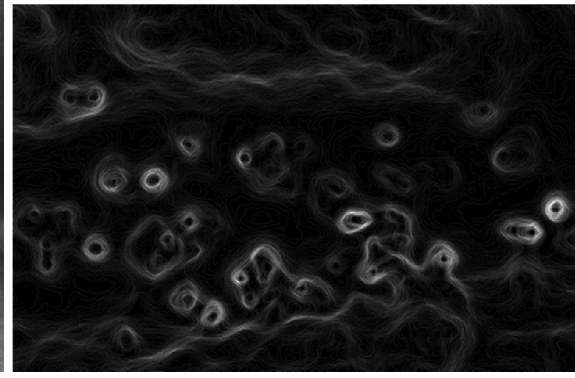

(b) magnitude of gradient

Fig. 1. Vessels captured in intravital microscopy. (a) Dark area corresponds to vessels and bright, roughly circular regions inside vessels are white blood cells. Note the high level of clutter due to the traveling, rolling, and stationary cells causing a challenge in automated segmentation as the gradient magnitude is very high near cell (b).

algorithm for the images captured by an intravital microscopy (IVM). The IVM is an in vivo imaging method that can capture both vasculature and flowing, rolling and stationary cells inside them. Unlike the retina images, the vessels from IVM can be highly cluttered by cells (Figure 1). These clutters result in high gradient around the boundary of cells (Figure 10) and its gradient vector flow (GVF) of pixels inside vessel to point toward a cell rather than the vessel boundary (Figure 2.b). Simple removal of the static cells using cell detection does not remedy the problem because the replacement pixels also cause an unwanted gradient to form around the detected cell boundaries.

In order to robustly guide segmentation toward the boundary of vessel even in the presence of a high level of cell clutter, we segment vessels with a set of virtual point pairs called "vessel probes" by applying forces including Vessel Vector Flow (VVF) which moves the vessel probes toward the vessel area while Vessel Boundary Vector Flow (VBVF) moves the probes to localize the boundary of vessels. The probes are moved according to Newtonian physics. By incorporating cell detection, the VVF and VBVF forces are computed to minimize the effect of gradient caused by the cells. Once the probes stabilize, they are connected to delineate the vessel boundary (Figure 2,f). Our method can segment vessels even when the cells [1] and vessels [2] are static making the temporal processing ineffective. The vessel probes are randomly initialized thus the method does not require initialization. We tested the method on a set of challenging real in vivo images of vessels in liver of mice cluttered with white blood cells and the result is encouraging.

\section{Previous Works}

A number of vessel segmentation algorithms has been published over the years. Kirbas and Quek [3] provide a thorough survey of the vessel segmentation methods. Majority of the work involve segmentation in retina images. The 


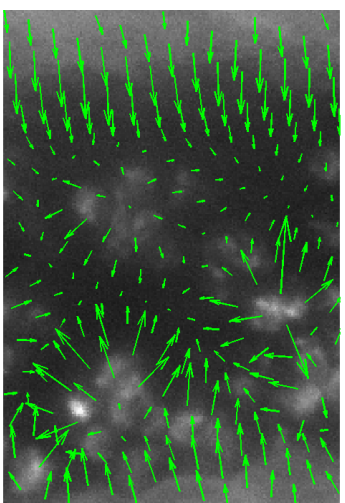

(a) intensity vector flow

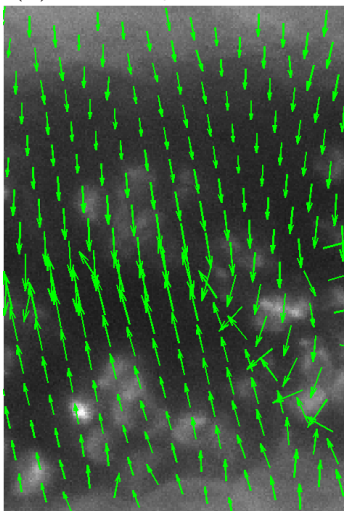

(d) vessel vector flow (VVF)

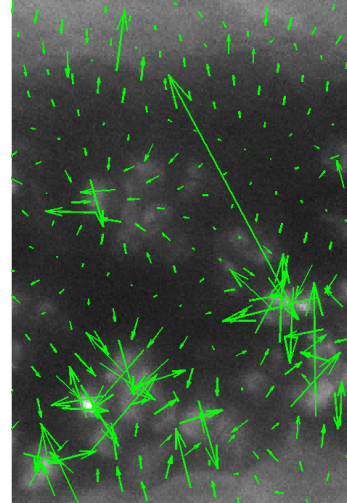

(b) gradient vector flow

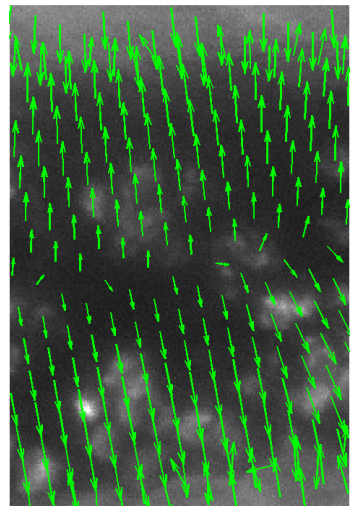

(e) vessel boundary vector flow (VBVF)

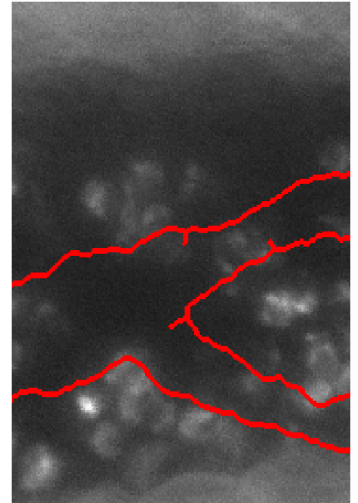

(c) without VVF and VBVF

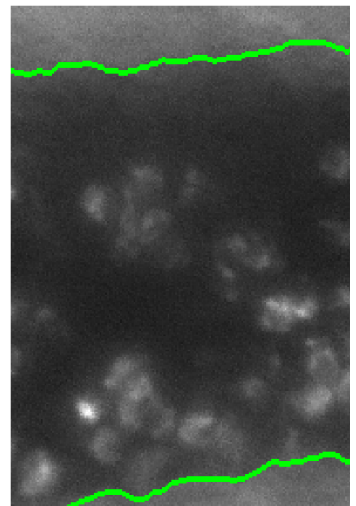

(f) our method

Fig. 2. Note how the intensity vector flow (which should point toward the higher intensity is corrupted by the presence of cell (a) while the vessel vector flow points toward the center of vessels. The gradient vector flow (b) is not a reliable for pointing toward the vessel boundary. The improvement in VVF (d) and VBVF (e) directing toward the center and boundary of vessel while avoiding the noise induced by cell clutter is achieved. The final segmentation are shown in (c) and (f) and demonstrates that our method can reliably segment vessels even with a presence of cells.

segmentation in presence of clutter and pathological diseases can be problematic. Shu et al. 4] segments retina vessels in disease conditions by discarding centerlines with gradient vector fields that were inconsistent with vessels. In our work, the clutter is inside vessels and frequently touching the vessel boundary causing noise in gradient vector flow along vessel boundaries (Figure 10).

Intravital microscopy (IVM) can capture both vessels and cells resulting in new challenges. An active contour based method was used to segment the boundary of the one large blood vessel of a cremaster muscle in a video sequence [2]. A sequential frame difference forms the initialization of the snake. A multi-scale 


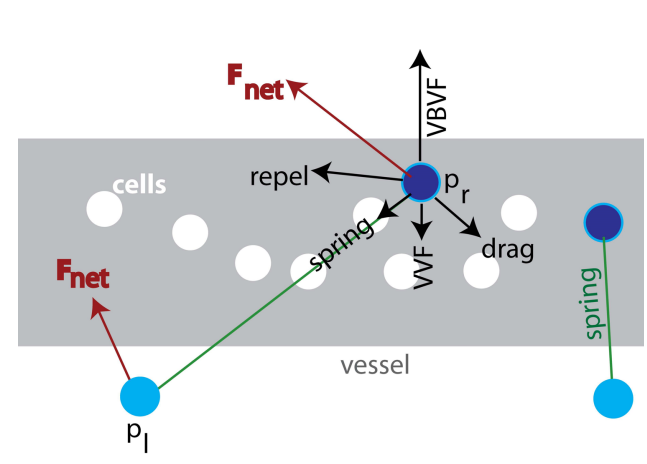

(a)

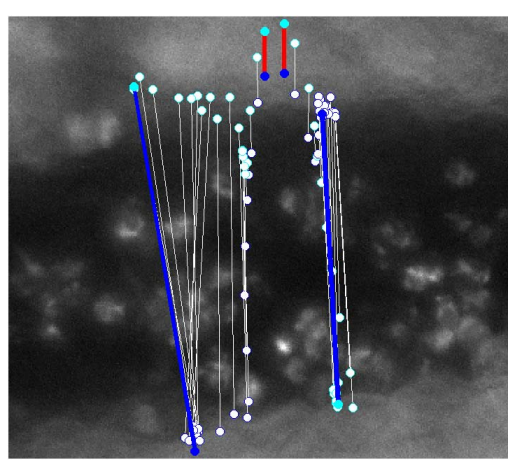

(b)

Fig. 3. Vessel Probe (left) and the visualization of their movement (right). Left: all forces are shown in black while the net force is draw in red. All forces are shown only for the left probe. Right: the iterations of the probes are shown. Probes are shown in red for initial orientation, blue at convergence, and white for all iterations in between.

approach was used to speed up the snake optimization, and gradient vector flow was used as external energy to be less resistant to noise. Also, multiple ribbon snakes [5] were used by [1] to segment the vasculature of the liver in IVM images after removing the moving cells using temporal median filtering. In our images, the vessel movement is minimal and a number of stationary cells is significant making the frame differencing ineffective for vessel localization initialization nor the removal of cells.

The 3-D vessel crawler by McIntosh and Hamarneh [6] uses a physics based model for segmenting 3-D vessels in MRA images. Points connected by springs crawl along the vessel. The crawler detects when it reaches a vessel junction and then spawns a new vessel crawler toward one of the uncrawled paths. Again, cells were not present in the vessels of images that were segmented.

\section{Method}

\subsection{Vessel Probe}

A vessel probe $(p)$ is constructed by two virtual points, $p_{l}$ and $p_{r}$, with an identical mass of $m_{p}$ (Figure 3.a). The probes are moved so that center of $p$ is inside vessel and $p_{l}$ and $p_{r}$ converges at the boundary of vessel. They are connected by a spring with a spring constant of $K_{s}$ and the equilibrium length $L$ which is the length of the spring at which the spring force is zero.

Initially, the virtual points of vessel probes are randomly positioned at $\boldsymbol{s}_{\boldsymbol{l}}$ and $s_{\boldsymbol{r}}$. As the same equation applies to the motion of either points, we will describe for one point and let $s$ be a location of a virtual point. The point's velocity $\boldsymbol{v}$ and acceleration $\boldsymbol{a}$ are initialized to be zero vectors. Each virtual point responds to the net force $\left(\boldsymbol{F}_{\boldsymbol{n e t}}\right)$ according to the Newtonian Physics. 
Table 1. Forces applied on Vessel Probes. $\boldsymbol{F}_{\boldsymbol{n} \text { et }}$ is the sum of all forces for each virtual point.

\begin{tabular}{|l|l|}
\hline Name & Task \\
\hline \hline Vessel Vector Flow (VVF) & moves the probe toward the vessel \\
\hline $\begin{array}{l}\text { Vessel Boundary Vector Flow } \\
\text { (VBVF) }\end{array}$ & $\begin{array}{l}\text { moves the virtual points to } \\
\text { localize on the vessel boundary }\end{array}$ \\
\hline Spring & $\begin{array}{l}\text { keeps two virtual points connected } \\
\text { within a reasonable length }\end{array}$ \\
\hline Repel & $\begin{array}{l}\text { spreads the probes to cover the vessel } \\
\text { with a smaller number of probes }\end{array}$ \\
\hline Drag & slows down the probes to converge at vessel \\
\hline
\end{tabular}

$\boldsymbol{F}_{\boldsymbol{n} \text { et }}$ is a sum of all forces applied on the probe. Forces are summarized on Table 1] Each virtual point is moved responding to $\boldsymbol{F}_{\boldsymbol{n} \text { et }}$ exerted for $T$ seconds. To simplify the implementation, we make two assumptions. First, the change in the forces during $T$ seconds due to the movement of points is not significant. Second, the direction and magnitude of force within a pixel is constant. We move each probe for $T$ seconds by breaking $T$ into smaller $\delta t$ where $\boldsymbol{F}_{\boldsymbol{n} \boldsymbol{e}}$ is constant (within a pixel). Note that position is calculated at a sub-pixel accuracy and the "pixel" refers to the area unit rather than a distance. The acceleration due to the force is $\boldsymbol{a}_{\boldsymbol{n e t}}=\boldsymbol{F}_{\boldsymbol{n e t}} / m_{p}$. At constant acceleration, the velocity after $\delta t$ would be $\boldsymbol{v}^{\prime}=\boldsymbol{v}+\boldsymbol{a}_{\boldsymbol{n} \boldsymbol{e t}} \delta t$. We compute the $\delta t$ using a quadratic formula, $\delta t=$ $\frac{-2 \boldsymbol{v} \pm \sqrt{(2 \boldsymbol{v})^{2}+8 \boldsymbol{a}(\hat{\boldsymbol{s}}-\boldsymbol{s})}}{2 \boldsymbol{a}}$ where $\hat{\boldsymbol{s}}$ is the intersection between the pixel boundary of $\boldsymbol{s}$ and the vector from $s$ moved along $\boldsymbol{v}$. The probe is moved to $\boldsymbol{s}^{\prime}=\boldsymbol{s}+\frac{1}{2}\left(\boldsymbol{v}^{\prime}+\boldsymbol{v}\right) \delta t$. Each probe is moved until the aggregate of $\delta t$ is $T$. A vessel probe convergence is found when the movement is minimal.

\subsection{Vessel Vector Flow Force}

The cells inside vessels cause significant noise in both the gradient magnitude (Figure 1,b) and the gradient vector flow (Figure 2.b). Note that the image gradient around cell boundary is significant and often stronger rather than around vessel. Even after a median filtering (with a kernel size of 15x15), the gradient magnitude around a vessel is still relatively weak. Most of the gradient vector flow [7] points toward cells rather than a vessel boundary which will make the segmentation based on gradient vector flow difficult. We propose Vessel Vector Flow (VVF) force that will robustly attract the center of vessel probes to the center of vessel while avoiding the distraction caused by cells cluttering vessel (Figure 1).

First, we detect cells by classifying each pixel as either cell or non-cell based on the radial mean. For each pixel, we compute the means of intensity of pixels within radius ranges of $[0,7]$ pixels $\left(\mu_{1}\right),[8,13]$ pixels $\left(\mu_{2}\right)$ and $[13,21]$ pixels $\left(\mu_{3}\right) . \mu_{1}, \mu_{2}$, and $\mu_{3}$ are normalized so that their sum $=1$. A pixel is classified as a cell when $\mu_{1}>T_{r}$. 
Second, we compute the direction of the vessel vector flow $\left(\boldsymbol{q}_{\boldsymbol{d i r}}\right)$ as a vector that will guide toward the center of vessel while avoiding the clutters caused by cells. Note that we are using the term "vector flow" as finding the vector of flow toward the higher value of a function similar to the method that computes the vector flow of gradient [7. Given $f$ to be the intensity of an image, we create an inverse of an image $g$ so that the flow direction will guide toward a vessel area which is often darker than tissue. Then we compute the vector flow of $g$ while minimizing the effect of cells by setting $\nabla g(x, y)=0$ for the locations at $(x, y)$ that has been classified as a cell pixel. To avoid the gradient near the boundary of cell, $\nabla g(x, y)$ for the locations within 5 pixels of cell pixels are also assigned the values of zero. The vector flow $\boldsymbol{q}_{\boldsymbol{d i r}}=(u(x, y), v(x, y))$ is that minimizes

$$
\xi=\iint \mu\left(u_{x}^{2}+u_{y}^{2}+v_{x}^{2}+v_{y}^{2}\right)+|\nabla g|^{2}\left|\boldsymbol{q}_{\text {dir }}-\nabla g\right|^{2} d x d y
$$

The normalized vector of $\boldsymbol{q}_{\boldsymbol{d i r}}(\boldsymbol{q} \hat{\boldsymbol{d i r}})$ is used as the direction component of the VVF force (Figure 2]d). Note that they mostly point toward the center of vessel right through the cells.

Third, we compute the magnitude of VVF. We use the gravitational model to formulate the forces that attracts the probes toward the vessel while repelling from each other. In that model, the magnitude of force should be at the maximum near the convergence. The magnitude of $\boldsymbol{q}_{\boldsymbol{d i r}}$ is highest near the vessel boundary. We noted that the $\boldsymbol{q}_{\boldsymbol{d i r}}$ often converges near the center of vessels. The convergence index [8] measures the level of convergences of vectors within a window. We computed $C_{q_{d i r}}$, the convergence of $\boldsymbol{q}_{\boldsymbol{d i r}}$. Then, the magnitude of vector flow of $C_{q_{d i r}}$ is computed as the magnitude of VVF force. The VVF force is computed by combining the magnitude and direction $(\boldsymbol{q} \hat{\boldsymbol{d i r}})$. The VVF force is visualized in Figure 2. d. Note that magnitude is generally higher near vessel center.

\subsection{Vessel Boundary Vector Flow Force}

The VVF attracts probes toward the vessel. We now derive Vessel Boundary Vector Flow (VBVF) force which will attract the points of vessel probes to the vessel boundaries. Because of the strong gradient around cell boundaries, the GVF inside a vessel often points toward cell boundaries rather than vessel boundaries (Figure 2 b). We noted the magnitude of $\boldsymbol{q}_{\boldsymbol{d i r}}$ is stronger near vessel boundaries. So, the vector flow of $\left\|\boldsymbol{q}_{\boldsymbol{d i r}}\right\|$ is computed as the Vessel Boundary Vector Flow (b) (Figure 2.e).

\subsection{Other Forces: Spring, Repel and Drag}

First, the spring force is used to keep points on the vessel probe from drifting too far from each other when the gradient is weak. The spring force is computed as $\boldsymbol{F}_{\boldsymbol{s}}=K_{s} \boldsymbol{d}_{\boldsymbol{s}}$ where $\boldsymbol{d}_{\boldsymbol{s}}$ is the displacement vector from a virtual point to the equilibrium location of spring. Second, to cover the vessel boundary with a minimum number of points, a repel force is designed to push the vessel probes from each other based on distance and orientation. The repel force exerted by 
probe $p^{i}$ to $p^{j}$ at distance $d$ is $\frac{m_{p} \cdot m_{p}}{d^{2}} \boldsymbol{g}$ where $\boldsymbol{g}$ is a normalized vector from $p^{i}$ to $p^{j}$. The repel force on $p^{i}$ is computed as the sum of forces from all other probes. Third, for the vessel probes to converge, a drag force is applied to each point on the vessel probe. It is computed as $-K_{d} \boldsymbol{v}$ where $K_{d}$ is the coefficient of drag.

\subsection{Post Processing for Forming Continuous Vessel Boundary}

After the vessel probes have converged, they are connected to form boundaries of the vessel. The probes' virtual points are connected if their orientation is nearly parallel and they are within a small distance. Then the locations along connection is fine-tuned to the greatest magnitude of Vessel Boundary Vector Flow within a small window.

\section{Results}

\subsection{Dataset}

Rats for all experimental groups were surgically prepared for intravital microscopy imaging as described in [9. The liver is exposed and setup on an inverted fluorescence Olympus microscope. Images were captured by a DAGEMTI SIT 66 camera at a rate of 30 frames per sec. The white blood cells were visualized using rhodamine.

\subsection{Evaluation}

The algorithm was tested on five real images of vessels with cell clutters. The results with and without VVF and VBVF are compared (Figure 4). For the cases without those forces, we have used intensity vector flow and GVF instead. First, the segmentation is closer to the vessel boundary and are less effected by the cells. In image 4, the gradient of the bottom left side of is just too weak.
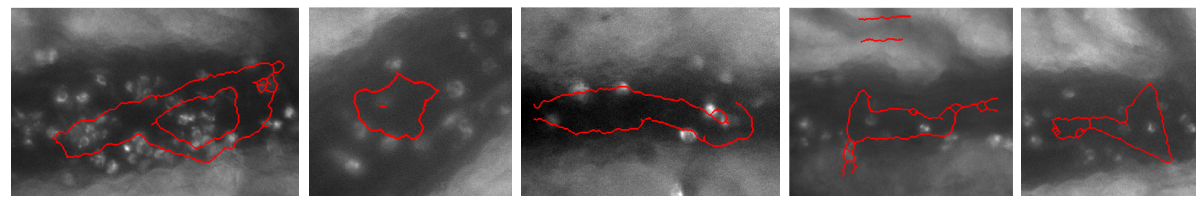

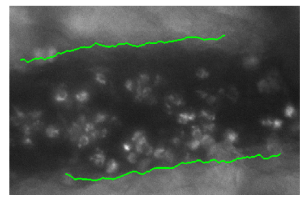

image 1

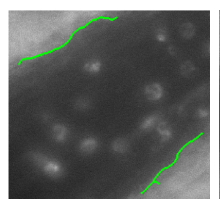

image 2

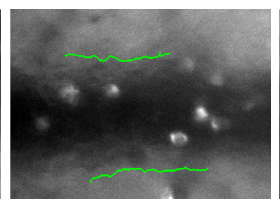

image 3

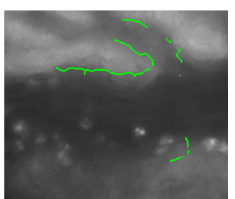

image 4

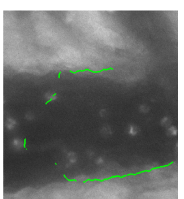

image 5

Fig. 4. Segmentation results on five real images . Top row includes results without using VVF or VBVF (using intensity vector flow and GVF instead) and the bottom row is our final results. 
To quantitatively assess the performance, the ground truth of the vessel boundary of those images was prepared by a biology technician. Note that the boundary is often very weak on gradient making the absolute pixel-level accurate ground truthing difficult. For each vessel boundary pixel segmented by the algorithm, the distance to the nearest vessel boundary marked in ground truth is computed. The root mean squared error (RMSE) of the distance for all machine segmented points are computed. On average, the RMSE of our method was $55 \%$ less than the method without VVF and VBVF.

\section{Conclusion}

We developed a vessel segmentation algorithm that is able to segment boundaries of vessels with a significant level of clutter due to presence of cells. These cells often divert the gradient vector flow toward the cells rather than the vessel boundary. Also, a number of cells are static, making the temporal differencing ineffective. We computed two forces, Vessel Vector Flow (VVF) and Vessel Boundary Vector Flow (VBVF), that guided the vessel probes and minimized the distraction caused by cells. A set of vessel probes were randomly deployed and moved according to the net of forces. The random deployment of probes eliminated the need for initialization. Comparing against the ground truth on five real in vivo images, the RMSE was $55 \%$ lower with addition of VVF and VBVF.

\section{References}

1. Schmugge, S., Kamoun, W., Villalobos, J., Clemens, M., Shin, M.: Segmentation of vasculature for intravital microscopy using bridging vessel snake. In: 3rd IEEE International Symposium on Biomedical Imaging: From Nano to Macro, pp. 177-180 (April 2006)

2. Tang, J., Acton, S.T.: Vessel boundary tracking for intravital microscopy via multiscale gradient vector flow snakes. IEEE Transactions on Biomedical Engineering 51(2), 316-324 (2004)

3. Kirbas, C., Quek, F.: A review of vessel extraction techniques and algorithms. ACM Computing Surveys (CSUR) 36(2), 81-121 (2004)

4. Shu, B., Lam, Y., Yan, H.: A novel vessel segmentation algorithm for pathological retina images based on the divergence of vector fields. IEEE Transactions on Medical Imaging 27(2), 237-246 (2008)

5. Mayer, H., Laptev, I., Baumgartner, A.: Multi-scale and snakes for automatic road extraction. In: Burkhardt, H.-J., Neumann, B. (eds.) ECCV 1998. LNCS, vol. 1406, pp. 720-734. Springer, Heidelberg (1998)

6. McIntosh, C., Hamarneh, G.: Vessel crawlers: 3d physically-based deformable organisms for vasculature segmentation and analysis. In: CVPR, vol. 1, pp. 1084-1091. IEEE, Los Alamitos (2006)

7. Xu, C., Prince, J.: Snakes, shapes, and gradient vector flow. IEEE Transactions on Image Processing 7(3), 359-369 (1998)

8. Kobatake, H., Hashimoto, S.: Convergence index filter for vector fields. IEEE Transactions on Image Processing 8(8), 1029-1038 (1999)

9. Clemens, M., Zhang, J.: Regulation of sinusoidal perfusion: in vivo methodology and control by endothelins. Seminars in Liver Disease 19(4), 383-396 (1999) 